\title{
COMMENT
}

\section{Only Time Will Tell: Cross-Sectional Studies Offer No Solution to the Age-Brain-Cognition Triangle: Comment on Salthouse (2011)}

\author{
Naftali Raz \\ Wayne State University
}

\author{
Ulman Lindenberger \\ Max Planck Institute for Human Development
}

\begin{abstract}
Salthouse (2011) critically reviewed cross-sectional and longitudinal relations among adult age, brain structure, and cognition $(\mathrm{ABC})$ and identified problems in interpretation of the extant literature. His review, however, missed several important points. First, there is enough disparity among the measures of brain structure and cognitive performance to question the uniformity of $\mathrm{B}$ and $\mathrm{C}$ vertices of the $\mathrm{ABC}$ triangle. Second, age differences and age changes in brain and cognition are often nonlinear. Third, variances and correlations among measures of brain and cognition frequently vary with age. Fourth, cross-sectional comparisons among competing models of ABC associations cannot disambiguate competing hypotheses about the structure and the range of directed and reciprocal relations between changes in brain and behavior. We offer the following conclusions, based on these observations. First, individual differences among younger adults are not useful for understanding the aging of brain and behavior. Second, only multivariate longitudinal studies, age-comparative experimental interventions, and a combination of the two will deliver us from the predicaments of the ABC triangle described by Salthouse. Mediation models of cross-sectional data represent age-related differences in target variables but fail to approximate time-dependent relations; thus, they do not elucidate the dimensions and dynamics of cognitive aging.
\end{abstract}

Keywords: brain, aging, cognition, longitudinal, mediation

The science which investigates causes is more instructive than one which does not, for it is those who tell us the causes of any particular thing who instruct us.

—Aristotle, Metaphysics

This much we know: With advancing age, the mammalian brain undergoes profound structural changes (for a review, see Raz \& Kennedy, 2009). The brain changes are accompanied by declines in cognitive performance that may be delayed by several decades (de Frias, Dixon, Fisher, \& Camicioli, 2007; Salthouse, 2009; Small, Dixon, \& McArdle, 2010). Numerous cross-sectional and a few longitudinal investigations have shown that the brains of older adults look and act differently from those of their younger counterparts (Hedden \& Gabrieli, 2004; Lindenberger, Burzynska, \& Nagel, in press; Nyberg et al., 2010; Raz, 2000; Raz et al., 2005) and that multiple measures of cognitive performance in many

Naftali Raz, Institute of Gerontology and Department of Psychology, Wayne State University; Ulman Lindenberger, Department of Developmental Psychology, Max Planck Institute for Human Development, Berlin, Germany.

This study was supported in part by National Institutes of Health Grant R37-AG-11230. The coauthors contributed equally to this comment.

Correspondence concerning this article should be addressed to Naftali Raz, Institute of Gerontology and Department of Psychology, Wayne State University, 87 East Ferry Street, 226 Knapp Building, Detroit, MI 48202, or to Ulman Lindenberger, Department of Developmental Psychology, Max Planck Institute for Human Development, Lentzeallee 94, D-14195 Berlin, Germany. E-mail: nraz@wayne.edu or lindenberger@mpib-berlin.mpg.de domains favor younger adults (Horn \& Cattell, 1967; Salthouse, 1996).

Based on such knowledge, it is natural to hypothesize meaningful relationships among age (A), brain (B), and cognition (C), and cognitive aging researchers have been working hard to elucidate these connections for the past two decades. In his review, Salthouse (2011) turned strong and harsh light onto the assembled findings. He forwent the nuances and illuminated an imbalance in the directionality assigned to the paths connecting the three nodes of the $\mathrm{ABC}$ triangle. With regard to the $\mathrm{BC}$ link, he saw little empirical justification for favoring one direction over the other. $\mathrm{He}$ also noted that the AB link may sometimes be absent altogether. In noting these empirical and conceptual flaws, Salthouse provided the researchers of cognitive aging with a much-needed reality check. Indeed, the days of simple linear truths, such as "bigger = better" and "the brain mediates between age and cognition," are over. Bigger brain volumes derived from MRI scans may reflect less than salubrious events, and the number of studies revealing negative associations between regional brain volumes and performance, although still not large, is growing steadily (Gautam, Cherbuin, Sachdev, Wen, \& Anstey, 2011; Salat, Kaye, \& Janowsky, 2002; Van Petten et al., 2004). As for associations between $\mathrm{B}$ and $\mathrm{C}$, the assumption that brain changes influence changes in cognition is highly plausible but not exclusive. The effect of the brain on cognition may be reciprocated, either through direct effects of training (e.g., Draganski et al., 2009), even in older adults (Lövdén et al., 2010, 2011), or via twice- or triceremoved causes such as lifestyle, nutrition, and health maintenance 
decisions mediated by cognitive factors (e.g., Hertzog, Kramer, Wilson, \& Lindenberger, 2009). Finally, some of the BC relations may lack directionality and be better understood as different manifestations of the influences exerted by other variables, not specified in the models. Salthouse's valuable message to those of us who are trying to navigate the deceptively simple yet devilishly complex terrain of cognitive neuroscience of aging is, things are not as bad as you think; they are much worse.

The message of Salthouse's (2011) review is clear, but by forgoing the examination of details and entertaining a bird's-eye view of the extant literature, Salthouse obscured the fact that the reviewed studies vary substantially in the ways they assess brain integrity. A cornucopia of methods-such as manual measures of regional volumes, estimation of local density and volume of gray and white matter derived from voxel-based morphometry, regional estimates of cortical thickness, semi-quantitative ratings of white matter hyperintensities (WMH) burden, measures of WMH volume, and indices of white matter organization derived from diffusion properties-were, at least conceptually, lumped together. Although Salthouse considered volume, WMH, and diffusionbased measures separately, he ignored significant differences among measurement methods within each domain. Methodological considerations and empirical evidence militate against the indiscriminate cataloging of diverse indicators under B. The analyses of MRI data generate multiple indices of brain integrity that not only are weakly correlated (Kennedy et al., 2009; Ziegler et al., 2010) but also differ in strength of their associations with age. For instance, age explains four times greater share of variance in $\mathrm{WMH}$ volumes than in WMH ratings (van den Heuvel et al., 2006); cortical thickness, volume, and cortical surface area appear quite independent and tap into different structural aspects of the aging brain (Lemaitre et al., 2010); individual differences in cortical thickness and volume stem from distinct genetic sources (Panizzon et al., 2009). Thus, B is not composed of uniform brain measures, as Salthouse presumed it to be.

We have similar reservations about the treatment of the $C$ vertex of the $\mathrm{ABC}$ triangle. An important problem in the extant $\mathrm{ABC}$ literature is that most of the reviewed studies address the issue with manifest variables (scores on cognitive tests or tasks) instead of latent variables (constructs based on multiple measures). A strong, often implicit assumption underlying this approach is that every cognitive test or task measures something specific and that its score reflects the integrity of a unique piece of brain circuitry. The likelihood of such a one-to-one mapping is very low. Often, it is a good idea to aggregate measures into meaningful constructs before exploring BC associations (Little, Lindenberger, \& Nesselroade, 1999; McArdle, 2009; Salthouse, 1991, 1996). Thus, ABC vertices are considerably fuzzier than Salthouse (2011) would have it, and, regrettably, this fuzziness was not addressed (or even mentioned) in the article.

\section{Three Questions}

Whereas we applaud the general objective of Salthouse (2011) to gain further insight into the nature of $\mathrm{ABC}$ relations, we want to elaborate on three issues, on which our views on how to approach this objective differ from his. The differences between approaches are more a matter of a degree than of kind. Nonetheless, we believe that these differences lead to divergent judgments about what can be learned about $\mathrm{ABC}$ relations from evidence that is restricted to samples of younger adults, findings from cross-sectional research designs, or both. Below, we discuss these issues and comment on the consequences of the noted discrepancies between approaches.

\section{Do Declines in Brain and Cognition Follow Linear Trajectories?}

Salthouse's review suggests that linear age trends generally offer a viable approximation to mean age differences and mean age changes in brain and behavior. For instance, Salthouse (2011) noted, based on analyses of his own data, that "additional analyses revealed that the quadratic age relations ... were not significantly different from zero.... In summary, nearly linear age-related declines in both cross-sectional and longitudinal comparisons have been reported in several major cognitive abilities" (pp. 754-755).

We remind that, in contrast to this view, nonlinear age trends in both $\mathrm{B}$ and $\mathrm{C}$ have been reported in studies with one or more suitable design characteristics, such as large sample size, large age range, and longitudinal observations (for a similar view, see Nilsson, Sternäng, Rönnlund, \& Nyberg, 2009). With respect to cognition, several studies have noted nonlinear age differences and age changes in one or more cognitive tests and abilities (e.g., de Frias et al., 2007; Lindenberger \& Ghisletta, 2009; Rönnlund, Nyberg, Bäckman, \& Nilsson, 2005; Schaie, 1996). In the Betula study data, for instance, longitudinal average decline has been found to be greater in the older age groups than in the younger age groups, defying the notion of linearity (e.g., de Frias et al., 2007; Nilsson et al., 2004).

Studies on age differences and age changes in brain structure also have documented a sizable amount of nonlinearity (e.g., Raz, Ghisletta, Rodrigue, Kennedy, \& Lindenberger, 2010; Raz et al., 2005). For instance, in a 5-year longitudinal study of 72 individuals age 20 to 77 years at baseline, we examined age-related differences and changes in 12 brain regions (Raz et al., 2005). We observed a highly differential pattern of regional age-related shrinkage. Although agerelated shrinkage observed in the prefrontal cortex was linear, declines in the hippocampal and the prefrontal white matter volumes increased with age. Moreover, late-onset, age-related increase in shrinkage was observed in the entorhinal cortex, a region that showed no shrinkage in the younger and the middle-aged participants. In the hippocampus, both linear and quadratic trends in age-related shrinkage were limited to hypertensive participants, a finding that highlights the importance of accounting for modifiers of age-related change. Notably, the extent of entorhinal cortex shrinkage was associated with memory performance and fluid intelligence at baseline (Raz et al., 2008; Rodrigue \& Raz, 2004). Thus, in contrast to Salthouse's (2011) assertion, aging of brain and aging of cognition are characterized by a substantial degree of nonlinearity. The causes of these nonlinearities are unclear, but our findings suggest that age-related changes in brain and cognition may vary as a function of age and may stem from the influence of age-related modifiers, such as elevated vascular risk.

\section{Are Variances and Covariances in Brain and Cognition Age Invariant?}

In addition to crowning linearity as a presumably sufficient descriptor of brain and cognitive aging, Salthouse (2011) asserted that the magnitude of individual differences generally does not 
vary as a function of adult age. For instance, summarizing the data from projects by Salthouse (2010a, 2010b) and Rönnlund et al. (2005), he concluded as follows: "Although age-related increases in between-person variability are sometimes found ... (e.g., Rabbitt, 1993) ... this is not the case in these data" (Salthouse, 2011, p. 754). Salthouse proceeded to generalize that "this finding is not specific to these two projects because nearly constant betweenperson variability has been found in cognitive variables from different standardized test batteries" (p. 755). He noted that his summary of results indicates "that not only is the magnitude of variability considerable in both the levels (cross-sectional) and the changes (longitudinal) in cognitive performance but that variability does not inevitably increase with advancing age" (p. 755). He further asserted that "there is no statistical reason why correlations involving cognitive variables would necessarily be weaker among young adults than among middle-aged or old adults" (Salthouse, 2011, p. 755).

We believe that, for methodological as well as empirical reasons, the claim that variances and covariances in measures of brain and cognition in adults do not depend on age is difficult to defend and is probably factually incorrect. First, assuming age-related stability of variances and covariances over the adult life span is tantamount to acceptance of the null hypothesis. Before making such a claim, one needs to ascertain the statistical power of detecting the differences in targeted outcomes. Monte Carlo simulations and formal analysis have demonstrated that the power to detect group differences in variances and covariances of change in typical longitudinal panel designs is distressingly low (Hertzog, Lindenberger, Ghisletta, \& von Oertzen, 2006, Hertzog, von Oertzen, Ghisletta, \& Lindenberger, 2008; von Oertzen, Hertzog, Lindenberger, \& Ghisletta, 2010), especially if the reliability of the measures is not close to perfect.

Second, empirical investigations conducted with appropriate statistical tools have revealed significant age differences in variances and covariances of cognitive and brain measures. In the realm of cognition, individual differences in rates of change for memory and verbal fluency were not reliably different from zero among the younger participants but emerged in the older age groups (e.g., de Frias et al., 2007). Similarly, relations between means and variances vary reliably between groups of younger and older adults as well as between individuals within age groups (Schmiedek, Lövdén, \& Lindenberger, 2009).

Age-related increments in individual differences in $\mathrm{B}$ and $\mathrm{C}$ reveal heterogeneity of individual trajectories of aging and may affect covariances within and between cognitive and brain-related variables. In fact, several studies found that correlations among multiple cognitive variables are higher among the older adults than in the younger age groups (Baltes \& Lindenberger, 1997; de Frias et al., 2007). As for age differences in BC relations, associations between hippocampal volume and age may be not reliably observed among the younger adults but emerge in the older age range (Raz, Gunning-Dixon, Head, Dupuis, \& Acker, 1998; Van Petten, 2004), and correlations between cortical thickness and executive functions can be larger in older than in younger adults (Burzynska et al., in press).

In summary, contrary to the assertion of Salthouse (2011), failure to detect reliable differences in variances or covariances in level and change may tell us less about their presence or absence than about the lack of statistical power. The identification of such differences with appropriate statistical tools in samples of adequate size supports the claim that individual differences in brain and behavior increase with advancing adult age (Lindenberger et al., in press).

\section{Does Falsification of a Cross-Sectional Mediation Model Constrain the Search Space of Longitudinal Relations Between Brain and Cognition?}

We agree with Salthouse's (2011) statement that longitudinal data are to be preferred to cross-sectional evidence for testing associations between changes in brain and cognition. At the same time, Salthouse argued that comparing competing models of $\mathrm{ABC}$ relations on the basis of cross-sectional data would help to reduce the number of viable causal models that can then be followed up by longitudinal evidence. For instance, he wrote that

it is important to emphasize that the results of these types of statistical control analyses do not provide a direct test of causal relations and instead merely provide an opportunity for implications of particular hypotheses of causal relations to be disconfirmed. However, because alternative models can often be postulated to account for relations among the variables, confidence in the plausibility of the hypothesized model can be increased if the data are found to be inconsistent with alternative models of the relations. (Salthouse, 2011, p. 757)

Alas, empirical findings and simulation research dash the hope of using cross-sectional findings in guiding longitudinal research. In a 5-year longitudinal study (Raz et al., 2005), we observed all kinds of relations between longitudinal and cross-sectional estimates of change. For some brain regions (the prefrontal cortex), cross-sectional trend provided a good estimate of the rate of change; for others (caudate nucleus and cerebellum), the crosssectional findings grossly underestimated the rate of shrinkage; and for still others (inferior parietal lobule), cross-sectional analyses missed reliable change revealed by longitudinal comparison.

In good agreement with this empirical evidence of diversity, formal analyses and Monte Carlo simulations have shown that the logic of using cross-sectional mediation models as an initial filter to weed out untenable assumptions about causal relations generally does not hold (e.g., Hofer, Flaherty, \& Hoffman, 2006; Lindenberger \& Pötter, 1998; Lindenberger, von Oertzen, Ghisletta, \& Hertzog, 2011; Maxwell \& Cole, 2007). The only exception emerges under highly restrictive conditions such as the "sequential ignorability assumption" (Imai, Keele, \& Tingsley, 2010), which is unlikely to be met in developmental research, in part for the reasons discussed above.

First, when a given cross-sectional mediation model fits the data well, there is no guarantee that the lead-lag relations of the corresponding longitudinal changes mimic the cross-sectional model. In fact, as Maxwell and Cole (2007) demonstrated, the crosssectional estimates are strongly biased for common models of change. Second, the situation is even worse: When a crosssectional mediation does not fit the data, a corresponding longitudinal model may still hold. Thus, although a cross-sectional mediation model provides a summary description of age-related and other individual differences, it does not qualify as a viable first step toward delineating the temporal ordering and causal structure of brain-related and behavioral changes (Lindenberger et al., 2011; Maxwell \& Cole, 2007). 
In this context, proportions of "explained age-related variance" obtained with cross-sectional mediation created confusion among cognitive aging researchers, especially if these proportions turned out to be impressively large. At the risk of being redundant, we can state that finding, in cross-sectional mediation, that a brain variable accounts for $70 \%, 80 \%$, or even $100 \%$ of the age-related variance in a cognitive variable leaves us with the following nonimplications. The finding does not imply that changes in the brain variable correlate with changes in the cognitive variable, that changes in the brain variable precede changes in the cognitive variable, or that changes in the brain variable cause changes in the cognitive variable. Furthermore, finding that the amount of age-related variance in a cognitive variable associated with a brain variable derived from cross-sectional mediation is zero does not imply the lack of association between changes in the brain and changes in cognition. It also does not imply that changes in the brain variable do not precede changes in the cognitive variable or that changes in the brain variable are functionally unrelated to changes in the cognitive variable. The only thing we can state with certainty about projecting from differences to change is that we do not know what is going on.

In summary, we agree that model comparisons based on crosssectional data are useful for explaining concurrent individual differences in brain and cognition. However, in contrast to Salthouse (2011), we propose that the cross-sectional approach is generally unsuitable for constraining the search space of longitudinal relations and should not be used for this purpose.

\section{Implications}

We have identified several empirical and methodological issues about which we disagree with Salthouse (2011). Aggregation of studies of brain structure under the rubric of B is unwarranted because of wide discrepancies in methods of measurement. Linear trends are insufficient to chart the aging of brain and cognition. Variances and covariances within and between brain-related and cognitive measures do change with adult age and tend to support the notion of heterogeneity in cognitive aging. Comparing different models of $\mathrm{ABC}$ relations based on cross-sectional data does not help in constraining the search for longitudinal relations between brain and cognition. Lumping together results from studies that use methods of discrepant reliability and validity jeopardizes metaanalytic interpretations of the findings.

Taken together, these claims have two important implications for the future study of brain-cognition relations in adulthood and old age. The first implication concerns the relative merit of studying samples of younger adults for understanding $\mathrm{ABC}$ relations. Indeed, if age trends were linear, if variances and covariances were age invariant, and if cross-sectional models were effective devices for constraining the search space of longitudinal $\mathrm{BC}$ relations, it would be reasonable to suggest that quite a lot about $\mathrm{ABC}$ relations can be learned by studying individual differences within samples of younger adults. If all three of these conditions were met, individual differences among younger adults would be likely to offer valid clues about individual differences that are driving age-related changes from early to late adulthood. However, given that the first two propositions are unlikely to be generally true and that the third proposition has been formally shown to be wrong, a research strategy focusing on younger adults to understand cogni- tive aging is likely to systematically miss its target. Studying the young is not a valid proxy or a sufficient basis for understanding the brain basis of cognitive aging.

The second implication is that the use of mediation analyses based on cross-sectional data, which is still common, should give way to longitudinal research designs. Longitudinal designs have some significant problems of their own, such as nonrandom attrition (e.g., Lindenberger, Singer, \& Baltes, 2002) and practice effects (e.g., Rabbitt, Diggle, Holland, \& McInnes, 2004; Salthouse, 1996); we need to direct our energy toward alleviating them. In addition, there is the fundamental question of choosing the optimal time window (cf. Maxwell \& Cole, 2007)— that is, deciding which sampling frequency faithfully captures the process of change in an observational study. The task of finding the correct sampling frequency is difficult enough for one variable (for discussion, see Raz et al., 2010), but it becomes increasingly complicated when the goal is to examine associations between two or more variables that change across time (e.g., B and C; cf. Boker, Molenaar, \& Nesselroade, 2009). Salthouse (2011) acknowledged some of these problems and stated the following:

In most cases a temporal lag probably exists between the changes in two sets of variables, and analyses of lagged changes are only meaningful if the longitudinal interval between observations matches the interval between early change in the presumed causal variable and later change in the presumed effect variable. (p. 757)

However, his pessimism about "these types of lead-lag analyses [being unable to] eliminate the problem if the total observation interval or the spacing of observations within the intervals does not match the timing of critical events" (p. 757) is not entirely warranted, given the existence of a sampling rate that can reproduce any given quasi-periodic process. Whereas the general trend of the developmental process may be monotonic, there may be significant periodicity around that trend, because at any time, the system parameters, cognitive and neural, are likely to represent a dynamic equilibrium of multiple factors (e.g., Boker et al., 2009; Raz et al., 2010). There may be a reason for the optimistic expectation that with advancement of our knowledge about neurobiological processes underpinning cognition in general and age-related change therein, the true shapes of age trajectories in $\mathrm{B}$ and $\mathrm{C}$, their between-person variation, and the range and direction of their interdependencies will emerge.

Particularly promising is the combination of longitudinal research design with intervention experiments (see Hertzog et al., 2009). Cognitive or fitness-related interventions attempt to alter brain functioning and boost cognitive performance. Hence, they provide more direct information on how changes in behavior are related to changes in the brain, including information about which brain mechanism support the maintenance of acquired skills over time.

\section{Conclusions}

Salthouse (2011) did the field a service by pointing out that focusing on one or two directed paths of the $\mathrm{ABC}$ triangle may lead to the unwarranted discounting of viable alternatives. We agree that all of us who have ever attempted a mediation analysis within the confines of a cross-sectional design (e.g., Lindenberger, Mayr, \& Kliegl, 1993; Raz et al., 1998) need to be reminded of 
how perilous this enterprise is. What Salthouse's analysis revealed is important, but what it failed to emphasize is vital: Even a perfect cross-sectional study is hopeless for the purpose of illuminating the causal structure of longitudinal change.

If cross-sectional designs are so hopeless, why do they remain so popular? Maxwell and Cole (2007) have asked that question. Their answer was that

little is known about the practical consequences of using crosssectional designs to study mediation ... [and] if differences between longitudinal and cross-sectional analyses of mediation are small in practice, researchers might be justified in continuing to study mediation with cross-sectional designs despite a theoretical disadvantage. (p. 24)

Unfortunately, the bias in cross-sectional mediation analyses of change is so substantial (Maxwell \& Cole, 2007) and the results so inconclusive (Lindenberger et al., 2011) that no practical reason can justify the continuation of business as usual.

We are not calling for abandonment of the cross-sectional studies of aging and development, as they provide valuable information about average age gradients, age differences in variances and covariances, and individual differences in general. However, the stated primary question of Salthouse's (2011) analysis - "the extent to which relations of age with measures of cognitive functioning are attributable to relations of age with measures of brain structure" (p. 753) - is unanswerable within the confines of crosssectional design. We agree with Salthouse that comparing competing models is a powerful method in science. Unfortunately, continuing use of this method in cross-sectional studies of aging and development will only perpetuate wandering among the vertices of the ABC Bermuda triangle, lest longitudinal assessments separated in time, experimental manipulation, or preferably both elucidate its causal dynamics.

\section{References}

Baltes, P. B., \& Lindenberger, U. (1997). Emergence of a powerful connection between sensory and cognitive functions across the adult life span: A new window to the study of cognitive aging? Psychology and Aging, 12, 12-21.

Boker, S. M., Molenaar, P., \& Nesselroade, J. R. (2009). Issues in intraindividual variability: Individual differences in equilibria and dynamics over multiple time scales. Psychology and Aging, 24, 858-862. doi: 10.1037/a0017912

Burzynska, A. Z., Nagel, I. E., Preuschhof, C., Gluth, S., Bäckman, L., Li, S.-C., ... Heekeren, H. R. (in press). Cortical thickness is linked to executive functioning in adulthood and aging. Human Brain Mapping.

de Frias, C. M., Dixon, R. A., Fisher, N., \& Camicioli, R. (2007). Interindividual variability in neurocognitive speed: A comparison of Parkinson's disease and normal older adults. Neuropsychologia, 45, 24992507.

Draganski, B., Gaser, C., Kempermann, G., Kuhn, H. G., Winkler, J., Büchel, C., \& May, A. (2009). Temporal and spatial dynamics of brain structure changes during extensive learning. Journal of Neuroscience, 26, 6314-6317.

Gautam, P., Cherbuin, N., Sachdev, P. S., Wen, W., \& Anstey, K. J. (2011). Relationships between cognitive function and frontal grey matter volumes and thickness in middle aged and early old-aged adults: The PATH Through Life Study. NeuroImage, 55, 845-855. doi:10.1016/ j.neuroimage.2011.01.015

Hedden, T., \& Gabrieli, J. D. (2004). Insights into the aging mind: A view from cognitive neuroscience. Nature Reviews Neuroscience, 5, 87-96.
Hertzog, C., Kramer, A. F., Wilson, R. S., \& Lindenberger, U. (2009). Enrichment effects on adult cognitive development: Can the functional capacity of older adults be preserved and enhanced? Psychological Science in the Public Interest, 9, 1-65.

Hertzog, C., Lindenberger, U., Ghisletta, P., \& von Oertzen, T. (2006). On the power of multivariate latent growth curve models to detect correlated change. Psychological Methods, 11, 244-252. doi:10.1037/1082989X.11.3.244

Hertzog, C., von Oertzen, T., Ghisletta, P., \& Lindenberger, U. (2008). Evaluating the power of latent growth curve models to detect individual differences in change. Structural Equation Modeling, 15, 541-563. doi:10.1080/10705510802338983

Hofer, S. M., Flaherty, B. P., \& Hoffman, L. (2006). Cross-sectional analysis of time-dependent data: Mean-induced association in ageheterogeneous samples and an alternative method based on sequential narrow age-cohort samples. Multivariate Behavioral Research, 41, 165187. doi:10.1207/s15327906mbr4102_4

Horn, J. L., \& Cattell, R. B. (1967). Age differences in fluid and crystallized intelligence. Acta Psychologica, 26, 107-129. doi:10.1016/00016918(67)90011-X

Imai, K., Keele, L., \& Tingsley, D. (2010). A general approach to causal mediation analysis. Psychological Methods, 15, 309-334. doi:10.1037/ a0020761

Kennedy, K. M., Erickson, K. I., Rodrigue, K. M., Voss, M. W., Colcombe, S. J., Kramer, A. F., . . Raz, N. (2009). Age-related differences in regional brain volumes: A comparison of optimized voxel-based morphometry to manual volumetry. Neurobiology of Aging, 30, 1657-1676. doi:10.1016/j.neurobiolaging.2007.12.020

Lemaitre, H., Goldman, A. L., Sambataro, F., Verchinski, B. A., MeyerLindenberg, A., Weinberger, D. R., \& Mattay, V. S. (2010). Normal age-related brain morphometric changes: Nonuniformity across cortical thickness, surface area and gray matter volume? Neurobiology of Aging. Advance online publication. doi:10.1016.j.neurobiolaging.2010.07.013

Lindenberger, U., Burzynska, A. Z., \& Nagel, I. E. (in press). Heterogeneity in frontal-lobe aging. In D. T. Stuss \& R. T. Knight (Eds.), Principles of frontal lobe functions (2nd ed.). New York, NY: Oxford University Press.

Lindenberger, U., \& Ghisletta, P. (2009). Cognitive and sensory declines in old age: Gauging the evidence for a common cause. Psychology and Aging, 24, 87-96.

Lindenberger, U., Mayr, U., \& Kliegl, R. (1993). Speed and intelligence in old age. Psychology and Aging, 8, 207-220. doi:10.1037/08827974.8.2.207

Lindenberger, U., von Oertzen, T., Ghisletta, P., \& Hertzog, C. (2011). Cross-sectional age variance extraction: What's change got to do with it? Psychology and Aging, 26, 34-47. doi:10.1037/a0020525

Lindenberger, U., \& Pötter, U. (1998). The complex nature of unique and shared effects in hierarchical linear regression: Implications for developmental psychology. Psychological Methods, 3, 218-230. doi:10.1037/ 1082-989X.3.2.218

Lindenberger, U., Singer, T., \& Baltes, P. B. (2002). Longitudinal selectivity in aging populations: Separating mortality-associated versus experimental components in the Berlin Aging Study (BASE). Journal of Gerontology: Series B: Psychological Sciences and Social Sciences, 57, P474-P482.

Little, T. D., Lindenberger, U., \& Nesselroade, J. R. (1999). On selecting indicators for multivariate measurement and modeling with latent variables: When "good" indicators are bad and "bad" indicators are good. Psychological Methods, 4, 192-211. doi:10.1037/1082-989X.4.2.192

Lövdén, M., Bodammer, N. C., Kühn, S., Kaufmann, J., Schütze, H., Tempelmann, C., ... Lindenberger, U. (2010). Experience-dependent plasticity of white-matter microstructure extends into old age. Neuropsychologia, 48, 3878-3883. doi:10.1016/j.neuropsychologia .2010 .08 .026 
Lövdén, M., Schaefer, S., Noack, H., Bodammer, N. C., Kühn, S., Heinze, H.-J., . . Lindenberger, U. (2011). Spatial navigation training protects the hippocampus against age-related changes during early and late adulthood. Neurobiology of Aging. Advance online publication. doi: 10.1016.j.neurobiolaging.2011.02.013

Maxwell, S. E., \& Cole, D. A. (2007). Bias in cross-sectional analyses of longitudinal mediation. Psychological Methods, 12, 23-44. doi:10.1037/ 1082-989X.12.1.23

McArdle, J. J. (2009). Latent variable modeling of differences and changes with longitudinal data. Annual Review of Psychology, 60, 577-605. doi:10.1146/annurev.psych.60.110707.163612

Nilsson, L.-G., Adolfsson, R., Bäckman, L., de Frias, C., Molander, B., \& Nyberg, L. (2004). Betula: A prospective cohort study on memory, health, and aging. Aging, Neuropsychology, and Cognition, 11, 134148. doi:10.1080/13825580490511026

Nilsson, L.-G., Sternäng, O., Rönnlund, M., \& Nyberg, L. (2009). Challenging the notion of early-onset cognitive decline. Neurobiology of Aging, 30, 521-524. doi:10.1016/j.neurobiolaging.2008.11.013

Nyberg, L., Salami, A., Andersson, M., Eriksson, J., Kalpouzos, G., Kauppi, K., . . Nilsson, L. G. (2010). Longitudinal evidence for diminished frontal cortex function in aging. Proceedings of the National Academy of Science, USA, 107, 22682-22686. doi:10.1073/ pnas. 1012651108

Panizzon, M. S., Fennema-Notestine, C., Eyler, L. T., Jernigan, T. L., Prom-Wormley, E., Neale, M., ... Kremen, W. S. (2009). Distinct genetic influences on cortical surface area and cortical thickness. Cerebral Cortex, 19, 2728-2735. doi:10.1093/cercor/bhp026

Rabbitt, P. (1993). Does it all go together when it goes? The Nineteenth Bartlett Memorial Lecture. Quarterly Journal of Experimental Psychology: Human Experimental Psychology, 46(A), 385-434.

Rabbitt, P., Diggle, P., Holland, F., \& McInnes, L. (2004). Practice and drop-out effects during a 17-year longitudinal study of cognitive aging. Journal of Gerontology: Series B: Psychological Sciences and Social Sciences, 59, P84-P97.

Raz, N. (2000). Aging of the brain and its impact on cognitive performance: Integration of structural and functional findings. In F. I. M. Craik $\&$ T. A. Salthouse (Eds.), Handbook of aging and cognition (2nd ed., pp. 1-90). Mahwah, NJ: Erlbaum.

Raz, N., Ghisletta, P., Rodrigue, K. M., Kennedy, K. M., \& Lindenberger, U. (2010). Trajectories of brain aging in middle-aged and older adults: Regional and individual differences. NeuroImage, 51, 501-511. doi: 10.1016/j.neuroimage.2010.03.020

Raz, N., Gunning-Dixon, F. M., Head, D. P., Dupuis, J. H., \& Acker, J. D. (1998). Neuroanatomical correlates of cognitive aging: Evidence from structural MRI. Neuropsychology, 12, 95-114. doi:10.1037/08944105.12 .1 .95

Raz, N., \& Kennedy, K. M. (2009). A systems approach to age-related change: Neuroanatomic changes, their modifiers, and cognitive correlates. In W. Jagust \& M. D'Esposito (Eds.), Imaging the aging brain (pp. 43-70). New York, NY: Oxford University Press

Raz, N., Lindenberger, U., Ghisletta, P., Rodrigue, K. M., Kennedy, K. M., \& Acker, J. D. (2008). Neuroanatomical correlates of fluid intelligence in healthy adults and persons with vascular risk factors. Cerebral Cortex, $18,718-726$.

Raz, N., Lindenberger, U., Rodrigue, K. M., Kennedy, K. M., Head, D., Williamson, A., ... Acker, J. D. (2005). Regional brain changes in aging healthy adults: General trends, individual differences and modifiers. Cerebral Cortex, 15, 1676-1689. doi:10.1093/cercor/bhi044

Rodrigue, K. M., \& Raz, N. (2004). Shrinkage of the entorhinal cortex over five years predicts memory performance in healthy adults. Journal of Neuroscience, 24, 956-963. doi:10.1523/JNEUROSCI.4166-03.2004

Rönnlund, M., Nyberg, L., Bäckman, L., \& Nilsson, L. G. (2005). Stability, growth, and decline in adult life span development of declarative memory: Cross-sectional and longitudinal data from a population-based study. Psychology and Aging, 20, 3-18.

Salat, D. H., Kaye, J. A., \& Janowsky, J. S. (2002). Greater orbital prefrontal volume selectively predicts worse working memory performance in older adults. Cerebral Cortex, 12, 494-505. doi:10.1093/ cercor/12.5.494

Salthouse, T. A. (1991). Theoretical perspectives in cognitive aging. Mahwah, NJ: Erlbaum.

Salthouse, T. A. (1996). Constraints on theories of cognitive aging. Psychonomic Bulletin \& Review, 3, 287-299. doi:10.3758/BF03210753

Salthouse, T. A. (2009). When does age-related cognitive decline begin? Neurobiology of Aging, 30, 507-514.

Salthouse, T. A. (2010a). Does the meaning of neurocognitive change change with age? Neuropsychology, 24, 273-278.

Salthouse, T. A. (2010b). Influence of age on practice effects in longitudinal neurocognitive change. Neuropsychology, 24, 563-572.

Salthouse, T. A. (2011). Neuroanatomical substrates of age-related cognitive decline. Psychological Bulletin, 137, 753-784. doi:10.1037/ a0023262

Schaie, K. W. (1996). Intellectual development in adulthood: The Seattle Longitudinal Study. New York, NY: Cambridge University Press.

Schmiedek, F., Lövdén, M., \& Lindenberger, U. (2009). On the relation of mean reaction time and intraindividual reaction time variability. Psychology and Aging, 24, 841-857. doi:10.1037/a0017799

Small, B. J., Dixon, R. A., \& McArdle, J. J. (2010). Tracking cognitionhealth changes from 55 to 95 years of age. Journal of Gerontology: Series B: Psychological Sciences and Social Sciences. Advance online publication. doi:10.1093/geronb/gbq093

van den Heuvel, D. M., ten Dam, V. H., de Craen, A. J., Admiraal-Behloul, F., van Es, A. C., Palm, W. M., ... van Buchem, M. A. (2006). Measuring longitudinal white matter changes: Comparison of a visual rating scale with a volumetric measurement. American Journal of Neuroradiology, 27, 875-878.

Van Petten, C. (2004). Relationship between hippocampal volume and memory ability in healthy individuals across the lifespan: Review and meta-analysis. Neuropsychologia, 42, 1394-1413. doi:10.1016/ j.neuropsychologia.2004.04.006

Van Petten, C., Plante, E., Davidson, P. S., Kuo, T. Y., Bajuscak, L., \& Glisky, E. L. (2004). Memory and executive function in older adults: Relationships with temporal and prefrontal gray matter volumes and white matter hyperintensities. Neuropsychologia, 42, 1313-1335. doi 10.1016/j.neuropsychologia.2004.02.009

von Oertzen, T., Hertzog, C., Lindenberger, U., \& Ghisletta, P. (2010). The effect of multiple indicators on the power to detect interindividual differences in change. British Journal of Mathematical and Statistical Psychology, 63, 627-646. doi:10.1348/000711010X486633

Ziegler, D. A., Piguet, O., Salat, D. H., Prince, K., Connally, E., \& Corkin, S. (2010). Cognition in healthy aging is related to regional white matter integrity, but not cortical thickness. Neurobiology of Aging, 31, 19121926. doi:10.1016/j.neurobiolaging.2008.10.015

Received May 19, 2011

Revision received May 19, 2011

Accepted May 23, 2011 